\title{
High Energy Beam THz Backward Wave Oscillator based on Double Corrugated Waveguide
}

\author{
Jun Zhang ${ }^{1}$ \\ Claudio Paoloni \\ Rosa Letizia \\ Lancaster University \\ Lancaster United Kingdom \\ LA1 4YR \\ ${ }^{1}$ National University of \\ Defense Technology \\ Changsha China
}

\author{
Neville C. Luhmann Jr \\ Branko Popovic \\ Logan Himes \\ Robert Barchfeld \\ Diana Gamzina \\ University of California Davis \\ Davis USA
}

\author{
Jinjun Feng \\ Li Li \\ Pan Pan \\ Ye Tang \\ Fuhzi Zhang \\ Beijing Vacuum Electronic \\ Research Institute and Vacuum \\ Electronics National Lab, China
}

\begin{abstract}
A$ new approach to realize $\mathrm{THz}$ BWOs relaxing the assembly challenge is presented. An international consortium including UC Davis, Beijing Vacuum Electronics Research Institute (BVERI), and Lancaster University is involved in the design and fabrication of $0.346 \mathrm{THz}$ BWOs to replace the bulky FIR laser at the plasma diagnostic at the NSTX-U fusion device. The use of a highly energetic beam permit and a wide channel, double corrugated waveguide permit to achieve about $4 \mathrm{~W}$ of output power at $0.346 \mathrm{THz}$.
\end{abstract}

Keywords: backward wave oscillator; double corrugated waveguide; electron beam; terahertz; millimeter waves

\section{Introduction}

Backward wave oscillators are an intrinsically low efficiency vacuum electron device [1]. The simple structure makes them among the most promising vacuum electron devices to generate power at $\mathrm{THz}$ frequency. The fabrication of BWOs in the $\mathrm{THz}$ regime, due to the small dimensions, is highly demanding [2 -3]. In particular, the alignment of the beam is very challenging due to the length of the interaction channel. An international consortium comprising UC Davis, Beijing Vacuum Electronics Research Institute (BVERI), and Lancaster University aims to find novel approaches to reduce the fabrication effort and the cost.

Different designs of $0.346 \mathrm{THz}$ BWOs based on the double corrugated waveguide (DCW) [4] were presented to replace the bulky FIR lased at the high-k plasma scattering diagnostic at the NSTX-U fusion device. About $0.5 \mathrm{~W}$ output power was achieved with a $13.8 \mathrm{kV}$ beam voltage, $10 \mathrm{~mA}$ beam current, and $100 \mu \mathrm{m}$ beam diameter. The beam tunnel was assumed $120 \mu \mathrm{m}$.

The DCW was demonstrated suitable to be fabricated by nano-CNC milling [3]. However, the beam alignment is very challenging, with high risk of low yield and beam interception with the pillars.

A new approach is proposed to relax the alignment difficulties and achieve a significantly higher output power. The use of a wide diameter, high energy beam permits one to decrease the filling factor thereby reducing the alignment effort.

\section{High energy beam BWO design}

The BWO in [5] was designed with a beam radius of 50 $\mu \mathrm{m}, 13 \mathrm{kV}$ and $10 \mathrm{~mA}$. The very narrow beam makes the alignment very challenging. A new BWO with a beam, with $80 \mu \mathrm{m}$ radius, $22 \mathrm{kV}$ of beam voltage, and $30 \mathrm{~mA}$ was designed while keeping the beam density almost the same (Table 1). The period of the DCW was also increased to match the synchronization between the phase velocity of the wave with the high energy beam. To accommodate the wider beam, the gap between pillars was set to $220 \mu \mathrm{m}$, one hundred microns wider than the $\mathrm{BWO}$ in [5].

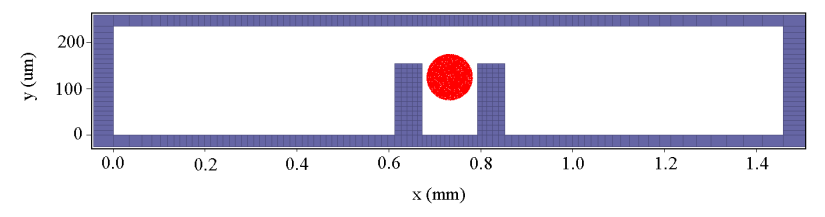

(a)

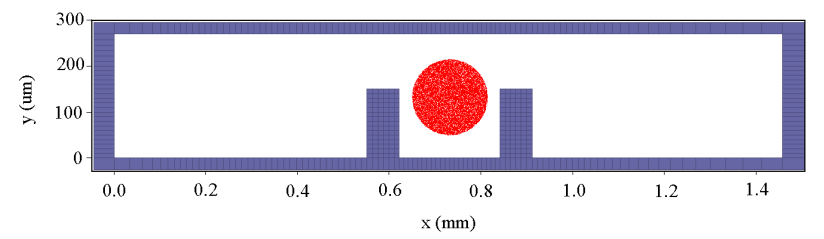

(b)

Fig.1 Cross section of beams and DCWs of the BWO [5] (a) and the new BWO (b)

This has allowed one to increase the distance from the edge of the beam to the pillar at $40 \mu \mathrm{m}$, in comparison to only $10 \mu \mathrm{m}$ in [5]. As a result, the beam alignment is expected to be much easier in the new BWO. However, the increased distance from the pillar wall makes the space charge field of the beam higher, so a higher guiding 
magnetic field is needed to prevent the diocotron instability of the cylindrical beam propagating through the rectangular waveguide.

Table 1. Dimensions of the BWOs

\begin{tabular}{|l|l|l|}
\hline & BWO [5] & New BWO \\
\hline Beam voltage $(\mathbf{k e V})$ & 12.8 & 22 \\
\hline Beam current $(\mathbf{m A})$ & 10 & 30 \\
\hline Magnetic field $(\mathbf{T})$ & 0.25 & 0.7 \\
\hline Beam radius $(\boldsymbol{\mu m})$ & 50 & 80 \\
\hline Pillar section $(\boldsymbol{\mu m})$ & $60 * 60$ & $70 * 70$ \\
\hline Beam channel $(\boldsymbol{\mu m})$ & 120 & 220 \\
\hline Pillar height $(\boldsymbol{\mu m})$ & 155 & 150 \\
\hline Period $(\boldsymbol{\mu m})$ & 140 & 170 \\
\hline Period number & 116 & 120 \\
\hline $\begin{array}{l}\text { Waveguide cross } \\
\text { section }(\boldsymbol{\mu m})\end{array}$ & $1500^{\star} 235$ & $1500^{\star} 270$ \\
\hline
\end{tabular}

The dispersion curve of the DCW computed by CSTMWS is shown in Fig.2, intersecting the beam line of 22 $\mathrm{keV}$ at the backward wave region at approximately 0.346 THz.

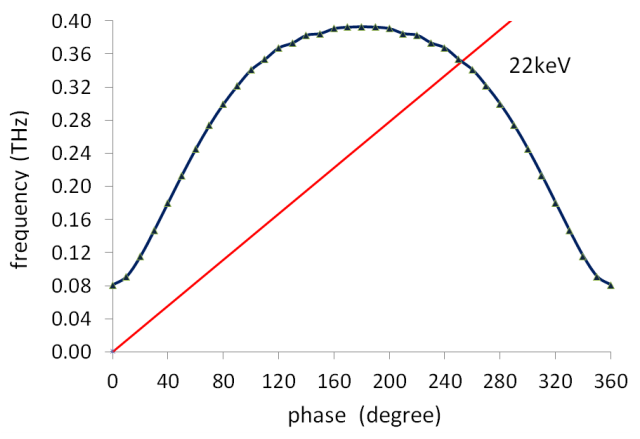

Fig.2 Dispersion curve

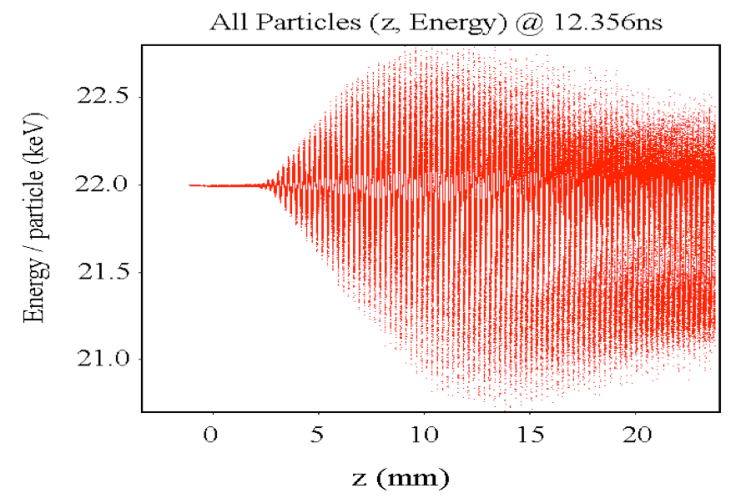

Fig.3 Beam energy after oscillation saturation
The new high energy beam $\mathrm{THz}$ BWO based on the DCW has been investigated by PIC simulations. Figure 3 presents the modulation and bunching of the beam. The optimized output power shown in Fig.4 is about $4.2 \mathrm{~W}$, with an efficiency of $0.64 \%$.

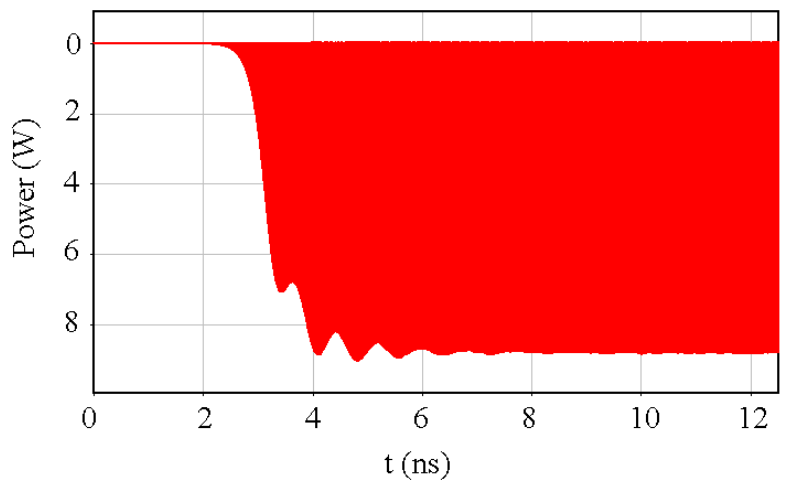

Fig.4 Output power

\section{Acknowledgements}

This work was supported by the UK EPSRC EP/L026597/1 grant, DARPA contract number G8U543366, NSF MRI grant CHE-1429258, DOE NSTX DE-FG02-99ER54518, DOD M67854-06-1-5118. ATK's Alliance Partnership Program, which was provided via the use of their MAGIC software. The Tesla K40 used for this research was donated by the NVIDIA Corporation.

\section{References}

1. M. Mineo, C. Paoloni, "Corrugated Rectangular Waveguide Tunable Backward Wave Oscillator for $\mathrm{THz}$ Applications", IEEE Trans. on Electron Devices, Vol.57, N.6, pp.1481-1484, June 2010.

2. A. Baig et al., "MEMS vacuum electronics," in Encyclopedia of Nanotechnology. Berlin, Germany: Springer-Verlag, 2012, pp. 1359-1368.

3. R. Barchfeld, D. Gamzina, A. Baig, L. R. Barnett, and N. C. Luhmann, "Nano CNC milling of two different designs of $0.22 \mathrm{THz}$ TWT circuits," in Vacuum Electronics Conference (IVEC), IEEE Thirteenth International, 2012, pp. 549-550.

4. M. Mineo and C. Paoloni, "Double-Corrugated Rectangular Waveguide Slow-Wave Structure for Terahertz Vacuum Devices," IEEE Trans on Electron Devices, pp. 3169-3175, Nov. 2010

5. Paoloni, C.; Lingna Yue; Xiaopin Tang; Zhang, F.; Popovic, B.; Himes, L.; Barchfeld, R.; Gamzina, D.; Letizia, R.; Mineo, M.; Luhmann, N.C., "THz backward-wave oscillators for plasma diagnostic in nuclear fusion," in IEEE International Conference on Plasma Sciences (ICOPS), 2015, 24-28 May 2015 